\title{
Femoral Tunnel Positioning in Anterior Cruciate Ligament Reconstruction: Anteromedial Portal versus Transtibial Technique-A Randomized Clinical Trial
}

\author{
Michele Venosa ${ }^{1}$ Marco Delcogliano ${ }^{2}$ Roberto Padua ${ }^{3}$ Federica Alviti ${ }^{4}$ Antonio Delcogliano ${ }^{1}$ \\ ${ }^{1}$ Department of Orthopaedic Surgery, San Carlo di Nancy Hospital - \\ GVM, Rome, Italy \\ ${ }^{2}$ Department of Orthopaedic Surgery, EOC - Ente Ospedaliero \\ Cantonale - Lugano, Switzerland \\ ${ }^{3}$ GLOBE, Evidence-based Orthopaedics Working Group of the Italian \\ Society of Orthopaedics and Traumatology; Nicola's Foundation, \\ Arezzo, Italy \\ ${ }^{4}$ Department of Physical Medicine and Rehabilitation, "La Sapienza" \\ University, Rome, Italy \\ Joints 2017;5:34-38. \\ Address for correspondence Michele Venosa, MD, San Carlo di Nancy \\ Hospital - GVM, Via Aurelia 275, 00165, Rome, Italy \\ (e-mail: michelevenosa@hotmail.com).
}

\author{
Abstract \\ Keywords \\ - anterior cruciate \\ ligament \\ - reconstruction \\ - femoral tunnel \\ - anteromedial portal \\ - transtibial \\ - computed \\ tomography
}

Purpose The purpose of this study was to investigate, through three-dimensional computed tomography (3D-CT), the accuracy of femoral tunnel positioning in patients undergoing anterior cruciate ligament (ACL) reconstruction, comparing transtibial (TT) and anteromedial (AM) techniques.

Methods We evaluated postoperative 3D-CT scans of 26 patients treated with ACL reconstruction with hamstrings autograft using a low accessory AM portal technique and 26 treated with the TT technique. The position of the femoral tunnel center was measured with the quadrant method.

Results Using quadrant method on CT scans, femoral tunnels were measured at a mean of 32.2 and $28.1 \%$ from the proximal condylar surface (parallel to Blumensaat line) and at a mean of 31.2 and $15.1 \%$ from the notch roof (perpendicular to Blumensaat line) for the AM and TT techniques, respectively.

Conclusion The AM portal technique provides more anatomical graft placement than TT techniques.

Level of Evidence Level I, randomized clinical study.

\section{Introduction}

Improper placement of bone tunnels is a major reason for anterior cruciate ligament (ACL) reconstruction failure. ${ }^{1-5}$ Several cadaveric and clinical studies have focused on the anatomical tunnel placement in ACL reconstruction to better restore normal knee kinematics and to improve rotatory stability and long-term outcome. ${ }^{6-9}$ Harner et $\mathrm{al}^{10}$ introduced the anteromedial (AM) portal technique for femoral tunneling to obtain a low-oblique drilling, which should be more anatomic than the traditional transtibial (TT) technique.

DOI http://dx.doi.org/ $10.1055 / \mathrm{s}-0037-1601413$ ISSN 2282-4324.
Different techniques were described to identify the center of femoral footprint, ${ }^{11-14}$ albeit most of the studies failed to adequately describe tunnel position. Kopf et a ${ }^{15}$ stated that three-dimensional computed tomography (3D-CT) is a reproducible and precise method to measure bone tunnel femoral position in ACL reconstruction. However, there is no clear information regarding 3D-CT evaluation of femoral tunnel position in the TT and AM portal techniques for femoral tunnel placement in ACL reconstruction.

The purpose of this study was to compare the position of femoral tunnel through 3D-CT in patients undergoing $\mathrm{ACL}$ 
reconstruction using the TT versus AM portal technique. The hypothesis of the study was that 3D-CT measurement of femoral tunnel position can reveal a significant difference between the AM and TT tunnel placement techniques.

\section{Methods}

We conducted a prospective randomized study to evaluate femoral tunnel position in patients undergoing ACL reconstruction with two different techniques (TT and AM techniques), with 3D-CT scan performed 20 days after surgery.

\section{Participants}

Between January 2012 and June 2014, 52 patients who were diagnosed with ACL rupture underwent primary ACL reconstruction using autologous hamstring tendon grafts. Diagnosis of ACL rupture was based on clinical diagnostic criteria summarized as follows: history of knee traumatic accident; subjective perception of knee laxity/instability; clinical evaluation (jerk test/pivot shift test, Lachman test); and magnetic resonance imaging (MRI) scan positive for complete ACL lesion.

Patients were randomly divided in two groups: 26 treated using an AM portal (group AM) and 26 using the TT technique (group TT). The randomization was performed by a statistical software before the beginning of the study. Group AM consisted of 20 men and 6 women with a mean age of 25.2 years (range: 16-40 years). Group TT consisted of 18 men and 8 women with a mean age of 26.4 years (range: 16-40 years). The surgery was performed on the right knee in 18 and 16 and on the left knee in 8 and 10 patients for groups AM and TT, respectively. The purpose of the study and the experimental procedures were explained to all the patients before they gave their written consent to participate.

\section{Surgical Technique}

In both the surgical procedures, the semitendinosus and gracilis tendons were harvested (from the affected limb) and prepared as a double-loop (four-stranded) graft.

In the TT ACL reconstruction, a tibial tunnel was created using an ACL tibial guide set at an angle of 55 degrees, with the tip of the tibial guide positioned at the central portion of the original ACL. The femoral tunnel was made using the TT technique at 1.30 to 2.00 o'clock position for the left knee and 10 to 10.30 o'clock position for the right knee.

In the AM ACL reconstruction, accurately placed portal was necessary. After formation of routine AM and AL portals, a central portal (through the fibers of the patellar tendon) and a low accessory AM portals were established (through arthroscopic view). The central portal is useful for the correct visualization of the femoral footprint; the low accessory AM portal is necessary to ensure adequate access for lateral tunnel drilling-since it lies close to the medial condyle, it is important to take attention to avoid iatrogenic cartilage damage. Using the lateral intercondylar ridge and the original femoral footprint as landmarks, we performed the femoral drilling.
In both groups, an EndoButton CL Ultra-Fixation (Smith \& Nephew, Andover, Massachusetts, United States) was used for femoral fixation; the grafts were pretensioned using a commercial tensiometer for 5 minutes on the tendon preparation board, followed by a maximal manual pull flexing and extending the knee through 15 cycles of full motion. Tibial fixation was granted by a bioabsorbable screw (BIORCI-HA, Smith \& Nephew) and a metallic staple.

\section{Outcome Measurements}

A postoperative 3D-CT was performed in all patients 20 days after surgery, obtaining femur and tibia reconstruction without soft tissue. Measurements were performed twice by an orthopaedic surgeon on two separate occasions to assess intraobserver reliability.

The femoral tunnel position was measured according to the quadrant method suggested by Bernard et al, ${ }^{14}$ obtaining a mediolateral view of the lateral femoral condyle in a strictly lateral position from the 3D-CT. The location of the tunnels was presented as the percentage distance from the intercondylar notch roof and quantified from the deepest subchondral contour to the center of the tunnel. The image was enclosed with a rectangular measurement frame (a $4 \times 4$ grid) formed by the Blumensaat's line, a parallel line tangent to the most inferior margin of the lateral condyle and two perpendicular lines tangent to the deepest/shallowest subchondral contour of the lateral femoral condyle.

The central point of the tunnel $(k)$ was calculated as $a / t$ and $b / h$, where $t$ is the total sagittal diameter of the lateral femoral condyle along Blumensaat's line, $a$ is the distance of $k$ from the deepest subchondral contour, $h$ is the maximum intercondylar notch height, and $b$ the distance of $k$ from Blumensaat's line. The ratios of $a / t$ and $b / h$ were expressed as percentage (-Fig. 1).

\section{Postoperative Treatment}

Both groups underwent the same standardized rehabilitation program with regular follow-ups. All patients were allowed for progressive weight-bearings with crutches (no brace). An early start to quadriceps exercises has been applied to improve early ROM development. With this protocol, patients gained full range of motion in 2 to 4 weeks, with an effective participation of the patient in the following rehabilitation phases. Balance and proprioception training, started early in the postoperative period, facilitate a positive effect on joint position sense, muscle strength, experienced knee function, and return to full activity. Closed chain exercises have been introduced in early rehabilitation, with benefits such as stimulation of proprioceptors, reduction of shear and acceleration forces, and development of dynamic knee stability; we consider closed-chain exercises safer and more functional than openchain exercises.

All patients were supervised two to three times a week to assure that the correct quality of performance and level of difficulty was achieved.

With this protocol, patients return to sport activities 6 months after surgery. 


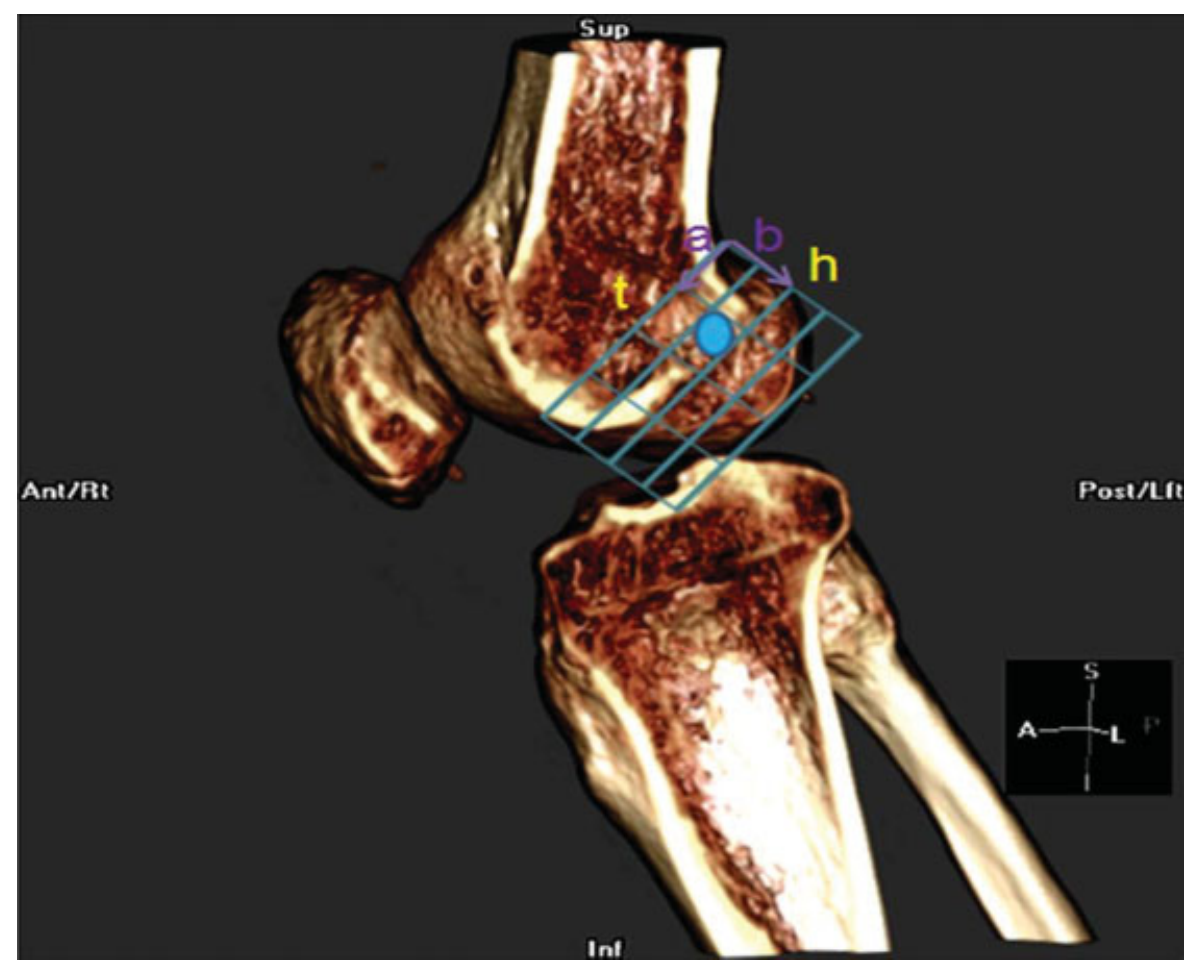

Fig. 1 Quadrant method according to Bernard et al. ${ }^{14}$ A rectangular $4 \times 4$ grid is drawn over the mediolateral view of the lateral femoral condyle in strictly sagittal sequence from three-dimensional computed tomography. $t$, Blumensaat's line; h, maximum intercondylar notch height; a, distance of femoral tunnel center from the deepest subchondral contour; $b$, distance of femoral tunnel center from Blumensaat's line.

\section{Data Analysis}

The sample size calculation was performed basing on the assumption that the main measurement (femoral tunnel position) is continuous and fairly normally distributed and that a $20 \%$ difference in the outcome measures is clinically relevant. With a significance level of $5 \%$, at least 20 participants per group were needed to be included.

Statistical analysis was performed using SPSS software (SPSS Inc., Chicago, Illinois, United States). $t$-tests were performed to compare TT and AM techniques tunnel position in $\mathrm{a} / \mathrm{t}$ and $\mathrm{b} / \mathrm{h}$ measures. To account for the comparisons of the femoral tunnels, the significance level was set at $p<0.001$. Data were presented as mean value \pm standard deviation.

\section{Results}

Measurement of femoral tunnel placement from the subchondral contour of the lateral femoral condyle $(a / t)$ was $32.2 \pm 3.3 \%$ and $28.1 \pm 1.6 \%$ for the AM and TT groups, respectively (-Fig. 2).

Measurement of femoral tunnel placement from the Blumensaat's line $(b / h)$ was $31.2+1.7 \%$ and $15.1+1.9 \%$ for the AM and TT groups, respectively ( - Fig. 3). Differences were significant in all comparisons $(p<0.0001)$.

\section{Discussion}

The purpose of this study was to evaluate femoral tunnel positioning in ACL reconstruction with 3D-CT using two different techniques (AM and TT techniques). Most studies

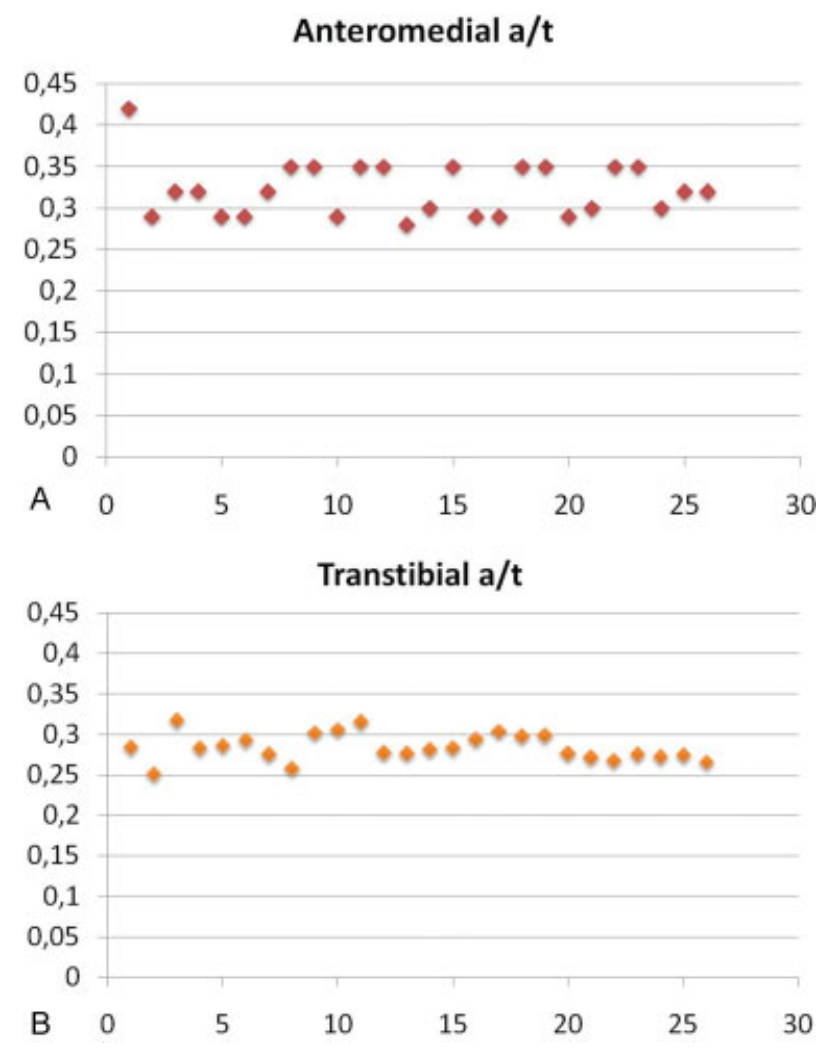

Fig. 2 Graph of $a / t$ measures in the anteromedial group (A) and in the transtibial group (B). 
Anteromedial b/h
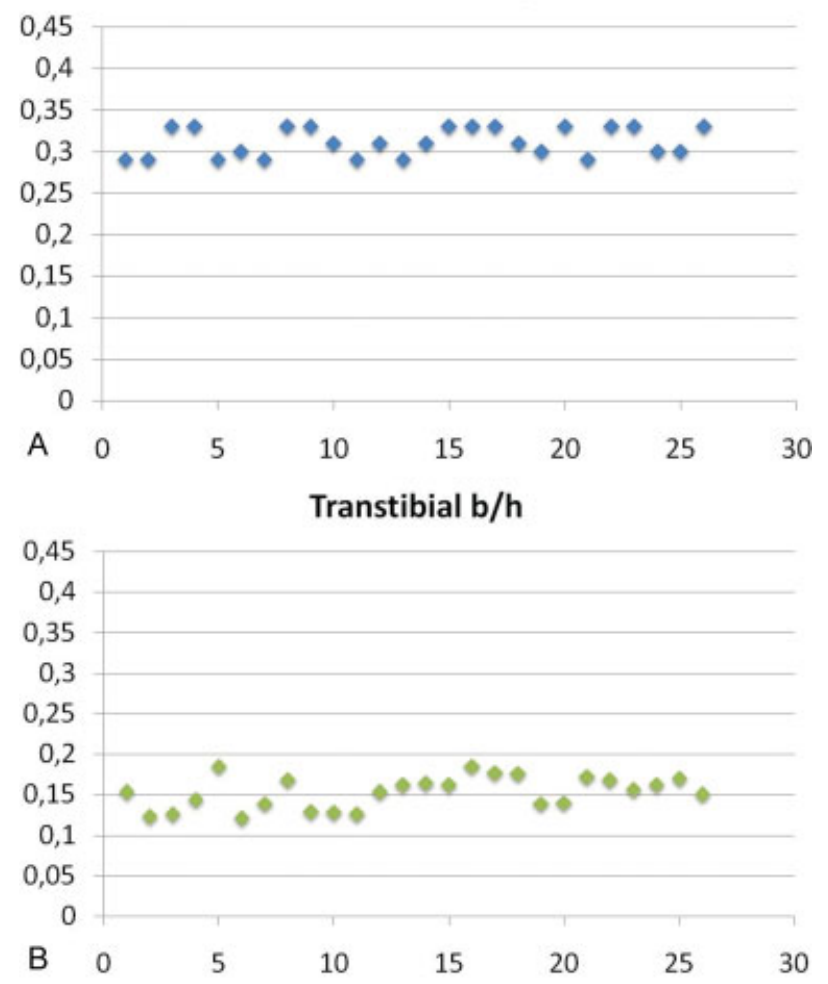

Fig. 3 Graph of $b / h$ measures in the anteromedial group $(A)$ and in the transtibial group (B).

have recently focused on the importance of restoring the anatomical characteristics of natural ACL; nonanatomical orientation of the graft can affect knee function with pain and instability, where a more vertical tunnel orientation can be correlated with rotatory instability, graft rupture, and poor outcomes. ${ }^{5,8,12,15-23}$ Techniques for restoring anatomical femoral footprint have been reported in AM reconstructions, such as out-in and in-out techniques. ${ }^{10,11,24-29}$ There are also previous studies claiming the plausibility of achieving anatomical femoral insertion site even using a TT technique. ${ }^{30}$ Heming ${ }^{31}$ commented that the tibial tunnel length must be shortened with a starting point closer to the joint line to obtain an accurate positioned footprint. Lee et $\mathrm{al}^{30}$ recently described a modified TT technique for single-bundle ACL reconstruction with the purpose of providing a more anatomical placement of the femoral tunnel.

Evaluating the tunnel position with traditional postoperative radiographs after ACL reconstruction is commonly known to be inaccurate because of the tunnel location within the 3D notch. ${ }^{32-34}$ Thanks to recent progresses in imaging tools and softwares, 3D-CT can provide high accuracy in visualization of bone structures. ${ }^{32-34}$ Three-dimensional assessment of the tunnel position gives accurate quantification of angles and diameters. Furthermore, the selective rotation of the model view (with the possibility of removing sections of bone) allows a clear visualization of regions traditionally difficult to see. ${ }^{32-34}$ In 2011, Meuffels et al ${ }^{32}$ compared plain radiographs, CT scans, and 3D reality imaging techniques, confirming that CT scans and 3D virtual reality images were more reliable than radiographs (higher intra- and interobserver agreement). ${ }^{34,35}$

The most common method used to assess femoral tunnel position is the quadrant method. Bernard et $\mathrm{al}^{14}$ reported that the center of the femoral insertion of ACL (in human cadaveric knees) was located at $24.8 \%$ of the distance $t$ measured from the deepest subchondral contour and at $28.5 \%$ of the height $h$ measured from Blumensaat's line. The values were 30.35 and $29.95 \%$, respectively, according to Tsukada et al, ${ }^{36} 26.9$ and $27.5 \%$, respectively, according to Steckel et al, ${ }^{37} 23.9$ and $37.95 \%$, respectively, according to Zantop et al, ${ }^{19,24} 27$ and $29 \%$, respectively, according to Yamamoto et al, ${ }^{17}$ and 26.6 and 30\%, respectively, according to Lee et al. ${ }^{16}$

The results of our study showed that $a / t$ was 32.2 and $28.1 \%$ whereas $b / h$ was 31.2 and $15.1 \%$ for the AM and TT techniques, respectively. These results confirm previous evaluations by other authors, ${ }^{18,25,38}$ who reported a higher or more anterior position than the natural ACL femoral footprint using a TT technique. Dargel et $\mathrm{al}^{39}$ reported suboptimal femoral tunnel position, whereas Giron et $\mathrm{a}^{40}$ reported the technical impossibility to restore femoral origin using a TT technique despite any modification as confirmed by Kopf et al. ${ }^{15,33}$

There are some limitations in this study. First, the role of the angle between the femoral tunnel and the graft was not taken into consideration. Second, no clinical outcomes were considered, such as stability and functional scores. Finally, the sample size was small.

In conclusion, the AM portal technique provides more anatomical graft placement than TT techniques in ACL reconstruction. This study provided analysis by 3D-CT models, a reproducible and precise method of measuring and demonstrating bone tunnel position.

\section{References}

1 Buss DD, Warren RF, Wickiewicz TL, Galinat BJ, Panariello R. Arthroscopically assisted reconstruction of the anterior cruciate ligament with use of autogenous patellar-ligament grafts. Results after twenty-four to forty-two months. J Bone Joint Surg Am 1993;75(09):1346-1355

2 Feller JA, Webster KE. A randomized comparison of patellar tendon and hamstring tendon anterior cruciate ligament reconstruction. Am J Sports Med 2003;31(04):564-573

3 Shelbourne KD, Nitz P. Accelerated rehabilitation after anterior cruciate ligament reconstruction. Am J Sports Med 1990;18(03): 292-299

4 Bowers AL, Bedi A, Lipman JD, et al. Comparison of anterior cruciate ligament tunnel position and graft obliquity with transtibial and anteromedial portal femoral tunnel reaming techniques using high-resolution magnetic resonance imaging. Arthroscopy 2011;27(11):1511-1522

5 Lee MC, Seong SC, Lee S, et al. Vertical femoral tunnel placement results in rotational knee laxity after anterior cruciate ligament reconstruction. Arthroscopy 2007;23(07):771-778

6 Woo SL, Wu C, Dede O, Vercillo F, Noorani S. Biomechanics and anterior cruciate ligament reconstruction. J Orthop Surg 2006; 1:2. Doi: $10.1186 / 1749-799 X-1-2$

7 Loh JC, Fukuda Y, Tsuda E, Steadman RJ, Fu FH, Woo SL. Knee stability and graft function following anterior cruciate ligament 
reconstruction: comparison between 11 o'clock and 10 o'clock femoral tunnel placement. 2002 Richard O'Connor Award paper. Arthroscopy 2003;19(03):297-304

8 Musahl V, Plakseychuk A, VanScyoc A, et al. Varying femoral tunnels between the anatomical footprint and isometric positions: effect on kinematics of the anterior cruciate ligamentreconstructed knee. Am J Sports Med 2005;33(05):712-718

9 Padua R, Alviti F, Venosa M, Mazzola C, Padua L. The influence of graft placement on clinical outcome in anterior cruciate ligament reconstruction. Joints 2016;4(01):12-16

10 Harner CD, Honkamp NJ, Ranawat AS. Anteromedial portal technique for creating the anterior cruciate ligament femoral tunnel. Arthroscopy 2008;24(01):113-115

11 van Eck CF, Lesniak BP, Schreiber VM, Fu FH. Anatomic single- and double-bundle anterior cruciate ligament reconstruction flowchart. Arthroscopy 2010;26(02):258-268

12 Yagi M, Wong EK, Kanamori A, Debski RE, Fu FH, Woo SL. Biomechanical analysis of an anatomic anterior cruciate ligament reconstruction. Am J Sports Med 2002;30(05):660-666

13 Gavriilidis I, Motsis EK, Pakos EE, Georgoulis AD, Mitsionis G, Xenakis TA. Transtibial versus anteromedial portal of the femoral tunnel in ACL reconstruction: a cadaveric study. Knee 2008; 15(05):364-367

14 Bernard M, Hertel P, Hornung H, Cierpinski T. Femoral insertion of the ACL. Radiographic quadrant method. Am J Knee Surg 1997; 10(01):14-21, discussion 21-22

15 Kopf S, Forsythe B, Wong AK, Tashman S, Irrgang JJ, Fu FH Transtibial ACL reconstruction technique fails to position drill tunnels anatomically in vivo 3D CT study. Knee Surg Sports Traumatol Arthrosc 2012;20(11):2200-2207

16 Lee KW, Hwang YS, Chi YJ, Yang DS, Kim HY, Choy WS. Anatomic single bundle anterior cruciate ligament reconstruction by low accessory anteromedial portal technique: an in vivo 3D CT study. Knee Surg Relat Res 2014;26(02):97-105

17 Yamamoto Y, Hsu WH, Woo SL, Van Scyoc AH, Takakura Y, Debski RE. Knee stability and graft function after anterior cruciate ligament reconstruction: a comparison of a lateral and an anatomical femoral tunnel placement. Am J Sports Med 2004;32(08): $1825-1832$

18 Kaseta MK, DeFrate LE, Charnock BL, Sullivan RT, Garrett WE Jr. Reconstruction technique affects femoral tunnel placement in ACL reconstruction. Clin Orthop Relat Res 2008;466(06): 1467-1474

19 Zantop T, Diermann N, Schumacher T, Schanz S, Fu FH, Petersen W. Anatomical and nonanatomical double-bundle anterior cruciate ligament reconstruction: importance of femoral tunnel location on knee kinematics. Am J Sports Med 2008;36(04): 678-685

20 van Eck CF, Kropf EJ, Romanowski JR, et al. Factors that influence the intra-articular rupture pattern of the ACL graft following single-bundle reconstruction. Knee Surg Sports Traumatol Arthrosc 2011;19(08):1243-1248

21 Kato Y, Maeyama A, Lertwanich P, et al. Biomechanical comparison of different graft positions for single-bundle anterior cruciate ligament reconstruction. Knee Surg Sports Traumatol Arthrosc 2013;21(04):816-823

22 Hosseini A, Lodhia P, Van de Velde SK, et al. Tunnel position and graft orientation in failed anterior cruciate ligament reconstruction: a clinical and imaging analysis. Int Orthop 2012;36(04):845-852

23 Chambat P, Guier C, Sonnery-Cottet B, Fayard JM, Thaunat M. The evolution of $A C L$ reconstruction over the last fifty years. Int Orthop 2013;37(02):181-186

24 Zantop T, Wellmann M, Fu FH, Petersen W. Tunnel positioning of anteromedial and posterolateral bundles in anatomic anterior cruciate ligament reconstruction: anatomic and radiographic findings. Am J Sports Med 2008;36(01):65-72
25 Abebe ES, Moorman CT III, Dziedzic TS, et al. Femoral tunnel placement during anterior cruciate ligament reconstruction: an in vivo imaging analysis comparing transtibial and 2-incision tibial tunnel-independent techniques. Am J Sports Med 2009; 37(10):1904-1911

26 Arnold MP, Duthon V, Neyret P, Hirschmann MT. Double incision iso-anatomical ACL reconstruction: the freedom to place the femoral tunnel within the anatomical attachment site without exception. Int Orthop 2013;37(02):247-251

27 Brown CH Jr, Spalding T, Robb C. Medial portal technique for single-bundle anatomical anterior cruciate ligament (ACL) reconstruction. Int Orthop 2013;37(02):253-269

28 Marcacci M, Zaffagnini S, Marcheggiani Muccioli GM, et al. Arthroscopic intra- and extra-articular anterior cruciate ligament reconstruction with gracilis and semitendinosus tendons: a review. Curr Rev Musculoskelet Med 2011;4(02):73-77

29 Pastrone A, Ferro A, Bruzzone M, et al. Anterior cruciate ligament reconstruction creating the femoral tunnel through the anteromedial portal. Surgical technique. Curr Rev Musculoskelet Med 2011;4(02):52-56

30 Lee SR, Jang HW, Lee DW, Nam SW, Ha JK, Kim JG. Evaluation of femoral tunnel positioning using 3-dimensional computed tomography and radiographs after single bundle anterior cruciate ligament reconstruction with modified transtibial technique. Clin Orthop Surg 2013;5(03):188-194

31 Heming JF, Rand J, Steiner ME. Anatomical limitations of transtibial drilling in anterior cruciate ligament reconstruction. Am J Sports Med 2007;35(10):1708-1715

32 Meuffels DE, Potters JW, Koning AH, Brown CH Jr, Verhaar JA, Reijman M. Visualization of postoperative anterior cruciate ligament reconstruction bone tunnels: reliability of standard radiographs, CT scans, and 3D virtual reality images. Acta Orthop 2011; 82(06):699-703

33 Kopf S, Forsythe B, Wong AK, et al. Nonanatomic tunnel position in traditional transtibial single-bundle anterior cruciate ligament reconstruction evaluated by three-dimensional computed tomography. J Bone Joint Surg Am 2010;92(06):1427-1431

34 Lertwanich P, Martins CA, Asai S, Ingham SJ, Smolinski P, Fu FH Anterior cruciate ligament tunnel position measurement reliability on 3-dimensional reconstructed computed tomography. Arthroscopy 2011;27(03):391-398

35 Forsythe B, Kopf S, Wong AK, et al. The location of femoral and tibial tunnels in anatomic double-bundle anterior cruciate ligament reconstruction analyzed by three-dimensional computed tomography models. J Bone Joint Surg Am 2010;92(06): $1418-1426$

36 Tsukada H, Ishibashi Y, Tsuda E, Fukuda A, Toh S. Anatomical analysis of the anterior cruciate ligament femoral and tibial footprints. J Orthop Sci 2008;13(02):122-129

37 Steckel H, Musahl V, Fu FH. The femoral insertions of the anteromedial and posterolateral bundles of the anterior cruciate ligament: a radiographic evaluation. Knee Surg Sports Traumatol Arthrosc 2010;18(01):52-55

38 Yang JH, Chang M, Kwak DS, Jang KM, Wang JH. In vivo threedimensional imaging analysis of femoral and tibial tunnel locations in single and double bundle anterior cruciate ligament reconstructions. Clin Orthop Surg 2014;6(01):32-42

39 Dargel J, Schmidt-Wiethoff R, Fischer S, Mader K, Koebke J, Schneider T. Femoral bone tunnel placement using the transtibial tunnel or the anteromedial portal in ACL reconstruction: a radiographic evaluation. Knee Surg Sports Traumatol Arthrosc 2009; 17(03):220-227

40 Giron F, Cuomo P, Edwards A, Bull AM, Amis AA, Aglietti P. Doublebundle "anatomic" anterior cruciate ligament reconstruction: a cadaveric study of tunnel positioning with a transtibial technique. Arthroscopy 2007;23(01):7-13 